\title{
Biomechanics of pelvic system: Towards a definition of the required mechanical properties of implants
}

\author{
M. Brieu ${ }^{1,2}$, M. Cosson ${ }^{3,4}$, P. Lecomte-Grosbras ${ }^{1,2}$, C. Rubod ${ }^{3,4}$ and J.-F. Witz ${ }^{2,5}$ \\ ${ }^{1}$ ECLille, LML, 59650 Villeneuve D’Ascq, France \\ 2 Univ Lille Nord de France, 59000 Lille, France \\ 3 Gynaecology Department, Jeanne de Flandre, CHRU, 59000 Lille, France \\ 4 INSERM U703, 59000 Lille, France \\ ${ }^{5}$ CNRS, UMR8107, 59650 Villeneuve D’Ascq, France
}

\begin{abstract}
Genital prolapse is a prevalent pelvic disorder inducing hypermobility of organs. Its physiopathology is not well understood as highlighted by the high rate of failure of the surgical treatments. A better definition of the pelvic tissues properties is needed to design more functional prostheses. Image registration is first used to describe the structure of the pelvic system. Experimental characterization is done to have a map of the mechanical properties of pelvic soft tissues and compare healthy and pathologic tissues behaviour. Then a model based on macromolecular approach and histologic composition is proposed.
\end{abstract}

\section{INTRODUCTION}

Pelvic mobility is essential to ensure the functionality of the pelvic organs. Reproduction, sexuality, and urinary or digestive functions are depending directly from the organs mobility. Genital prolapse is a prevalent pelvic disorder inducing hypermobility of pelvic organs. This pathology affects 1 of 3 women all ages combined, and more than $60 \%$ of patients older than 60 years $[1,2]$. The treatment is essentially surgical and generally consists in insertion of prosthetic material to restore functional mobility of organ. Many corrective surgical techniques have been developed, but all have a rate of failure close to $30 \%$. The surgery consists generally in introducing the prosthetic material into the vesicovaginal and rectovaginal spaces, but mechanical properties of such tissues are not well known. Moreover, their mechanical properties are strongly depending on their structure and histologic composition [3,4]. A better definition of the mechanical properties as well as the structure of pelvic organs is needed. It should help on the one hand to better understand the physiopathology of genital prolapse and on the other hand to design more functional prostheses.

With this objective, the mobility of organs have been studied using image registration technique, mechanical properties of pelvic tissues have been performed influence of age and pathology studied. A hyper-elastic model based on physical description and histological composition of tissue is then proposed.

\section{PELVIC MOBILITY CHARACTERIZATION}

The pelvic mobility is due to the mechanical properties of the pelvic organs on the one hand and the way they are arranged and linked together. Indeed the pelvic organs are support by a network of connective tissue organized in plane diffuse structure called fascia, or in dense structure called ligaments. To characterize the pelvic mobility, localize ligaments and fascia and understand the way organs are linked together dynamic Magnetic Resonance Images (MRI) have been first analysed. This technique is classically used for diagnostic of genital prolapse and consists in asking the patient to strain and acquiring 2D images in the sagittal plane all along the straining in order to observe organs motions. The figure 1 shows images taken at different stage of the straining on control patient without genital prolapse and patient with major prolapse with exteriorization of the uterus.

The images have been analysed by image registration by comparing the images of the MRI sequence to the initial one using a metric based on pixel intensities [5]. This method allows measuring the displacements on the contour of the four pelvic organs, Vagina (V), Uterus (U), Bladder (B) and Rectum (R). These results show areas with null displacement highlighting attachment of bladder and rectum respectively to the pubis and sacrum. It also highlights areas having same displacement showing the presence of fascia linking bladder and rectum to vagina. These fascias have a very important role because the surgery consists generally in inserting the prosthetic material in such areas.

\section{MECHANICAL CHARACTERIZATION}

Mobility is due to structural arrangement of the system and mechanical properties of tissues. Experimental characterization of the mechanical behaviour of organs and ligaments has been performed. First an experimental protocol has been defined allowing having reproducible results [6]. Vagina, rectum and bladder as well as three pairs of ligaments have been characterized on 45 cadavers. This allows having the map of the mechanical properties of pelvic system as shown on figure 2 .

Pelvic organs have a visco-hyper-elastic damageable behaviour. Figure 2 shows that vagina is the stiffer and seems to have the major sustaining role and ligaments are stiffer than organs. As Prolapse affects old patients, the 


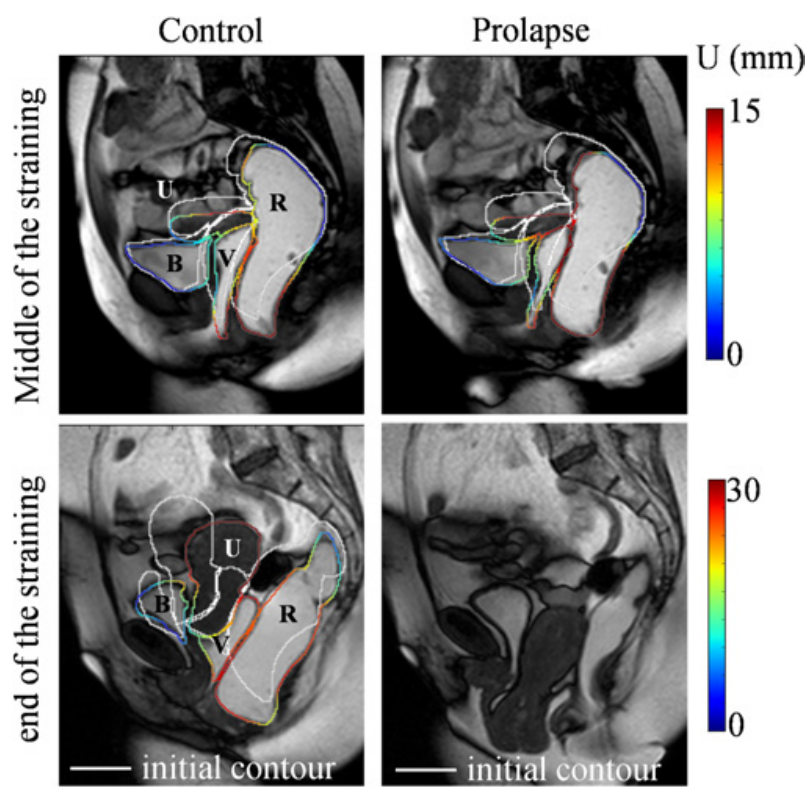

Figure 1. Displacement magnitude measured on dynamic MRI on control patient and patient with major prolapse.

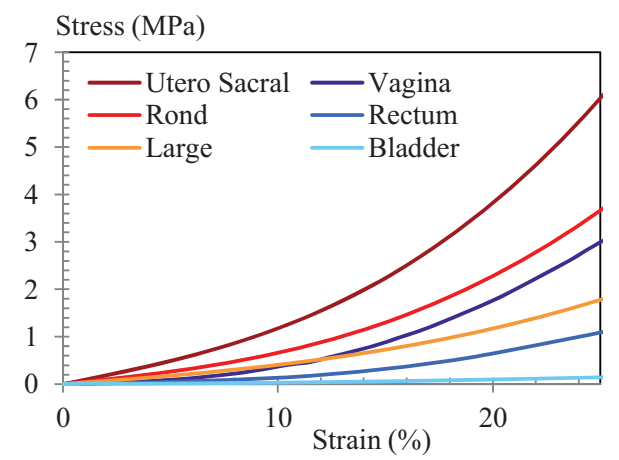

Figure 2. Displacement magnitude measured on dynamic MRI on control patient and patient with major prolapse.

influences of age and pathology on mechanical response of pelvic tissue have been studied, as shown on figure 3 .

Figure 3 shows that the rigidity of organs and ligaments increases with age as well as with the pathology. It is known that the mechanical properties of biological tissues depend on their structure and histologic composition. Tissues are indeed mainly composed of elastin and collagen. Collagen fibres are known to have high rigidity compare to elastin [7]. Elastin gives the deformability to the tissue but is synthetized only until puberty. Thus, the volume fraction of elastin decrease and tissues become more rigid with age as experimentally highlighted.

\section{HISTOLOGIC MODELLING OF TISSUES}

As mechanical behaviour of pelvic tissues is highly depending on the histological composition it is more relevant to define a model based on these physical observations. A hyper-elastic model based on macromolecular approach and elastin and collagen volume fraction has been introduced [8]. The stress-stretch relation is done through the derivation of energy density $W$ that depends on the length and the rigidity of the polymer
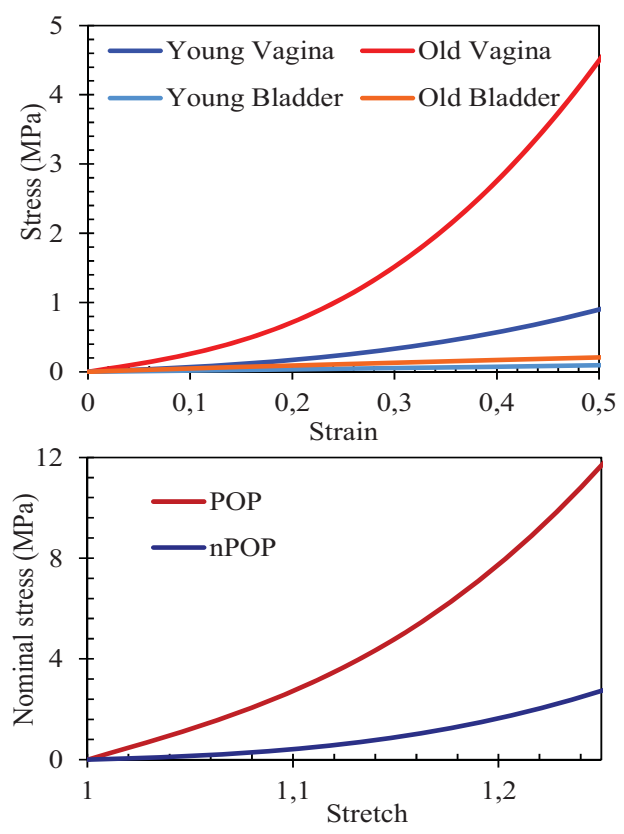

Figure 3. Influence of age (up) and pathology (down) on mechanical properties.

fibers. We define $W$ as a function of two energy densities, one for elastin and one for collagen fibres linked by a single parameter $\varphi$ corresponding to collagen volume fraction.

$$
\mathcal{W}(\underline{\underline{F}})=\phi \cdot \mathcal{W}^{\mathrm{c}}(\underline{\underline{F}})+(1-\phi) \cdot \mathcal{W}^{\mathrm{e}}(\underline{\underline{F}}) .
$$

The main hypothesis of this model is that the difference of mechanical properties is only a function of the collagen volume fraction. Some histologic characterizations have been performed on specimen mechanically characterized allowing to define the mechanical behaviour of elastin and collagen. This model allows having a hyper-elastic model of mechanical behaviour of pelvic soft tissue depending on a single parameter. An experimental campaign is currently done with parallel histological and mechanical characterization to validate the model.

\section{CONCLUSION}

The physiopathology of genital prolapse is very complex and not well understood as highlighted by the high rate of failure of all the surgical treatment. The objective of this work was to characterize the mobility of the pelvic system and to characterize the mechanical properties of the involved soft connective tissues. Thanks to the definition of an experimental protocol, characterization of pelvic organs and ligaments have been done. The increase of rigidity of the tissue with age and pathology has been highlighted. A model based on a macromolecular description and histological composition has been proposed allowing having a hyper-elastic model depending on only a single parameter. These results suggest that the use of the same prosthetic material for different anatomic localization, as it is actually done, might not be appropriate considering the highlighted difference in mechanical properties and histologic constitution of tissues. These results will lead 
to define more functional prosthetic materials and more targeted surgical strategy.

\section{References}

[1] Swift. S.E., Am. J. Obs. \& Gyn, 2000;183,:277-285

[2] WeberA et al, Am. J. Obs.\& Gyn, 2001;185:12991304
[3] Albert B. et al, Molecular Biology of the Cell. 2002 Garland Science. New York.

[4] Fung, Y.C., Biorheology of soft tissues. Biorheology 1973, 10,199-212.

[5] S. Klein et al, IEEE Trans. Im. Proc. 2007;16 :2879_ 2890.

[6] Rubod C et al. J Urol. 2007;178:320-325.

[7] Shen Z.L., et al., Biophy. J., 2008, 95:3956-3963.

[8] Gillibert J., et al Int. Uro Gyn. Ass. Conf., 2011, 